\title{
Total parenteral nutrition in a busy English district general hospital
}

\author{
I. M. Patanwala and J. M. Sayer \\ Gastroenterology, Doncaster Royal Infirmary, Doncaster, UK
}

Total parenteral nutrition (TPN) is an important adjunct in critically ill patients without an accessible or functional gut mucosa. It helps wound healing, builds resistance to infection and decreases length of stay in hospital. Decision to start TPN is complex, representing a balance between nutrition benefit, serious complications and high costs.

We analyzed and compared the use of TPN in an 800-bedded general hospital with national guidelines ${ }^{(1)}$ and also, the use of TPN in other centres internationally. A retrospective case-note review of all patients receiving TPN in the first 5 months of 2006 yielding data on demographics, area of use, duration and initiator was collected. More detailed analyses of indications, type of venous access, dietitian review relative to starting, reasons for stopping, complications and outcomes were collected for a subset of these patients:

\section{Duration of TPN}

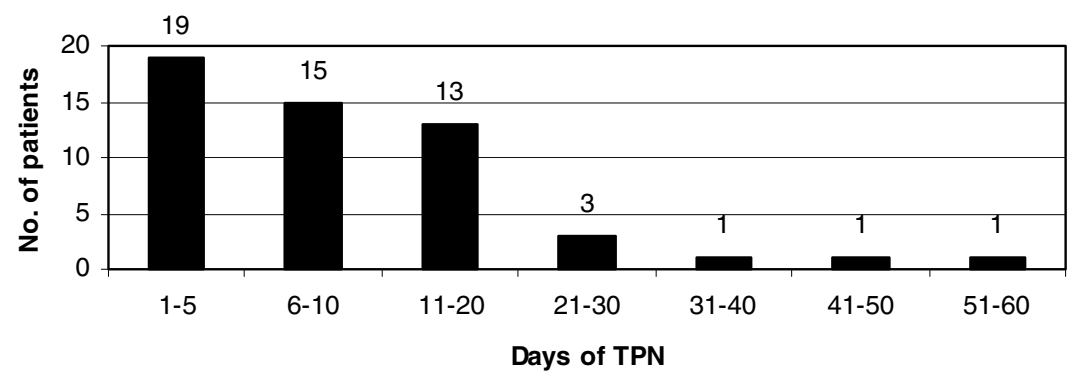

\section{Complications of TPN}

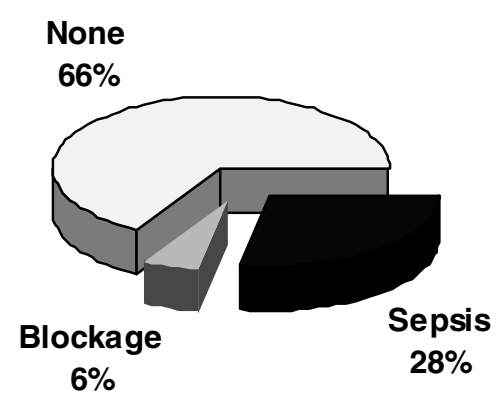

Fifty-three patients received TPN in 5 months with a median age of 61 (range 19-90) years of which $60 \%$ were males; $94 \%$ were surgical patients and the remaining medical. TPN was initiated on intensive/high dependency care in thirty-seven (70\%) patients and on the wards in the rest. Decision to start TPN was shared equally between intensivists and surgeons. No physician initiated TPN. Indications for TPN were: failed/non functional gut (39\%), inaccessible gut (28\%), unsafe enteral route (17\%) and gut perforation (16\%). Neoplastic bowel disease was present in $33 \%$. Central un-tunnelled venous access was most commonly used (67\%), as compared to tunnelled central lines $(17 \%)$, peripheral venous access $(11 \%)$ and peripherally inserted central catheter (PICC) lines (5\%). The median duration of TPN prescription was $8 \mathrm{~d}$ (range 2-54). A total of $12 \%$ patients did not get reviewed by a dietician until after their TPN was stopped. Complications included sepsis $(28 \%)$ and blockage $(6 \%)$ and were noted in those with central venous access. Commonest causes for stopping TPN were availability of enteral route $(78 \%)$ and line blockage or removal $(17 \%)$. The median stay of these patients in hospital was $55 \mathrm{~d}$ (range 16-98) at the end of which $89 \%$ were discharged home. A total of $11 \%$ patients died but the cause of death was not directly related to TPN or its complications. The cost of preparation of each bag of TPN was estimated at $£ 53.00$ without the addition of adjuncts such as glutamine.

TPN use in our hospital was comparable to other centres in Europe ${ }^{(2,3)}$, Asia ${ }^{(4)}$ and Australia ${ }^{(5)}$ except for higher rates of septic complications and lower rates of dietician involvement. Establishment of a multidisciplinary nutrition team by implementing the current national guidelines and using the data gathered above as impetus for change would help improve parenteral nutrition services and decrease complications in our hospital.

1. Stroud M (chair, guideline development group) et al. Nutrition support in Adults. (2006) Chapter 10: 192-206.

2. Alcazar V et al. (1998) Nutricion Hospitalaria, Spain 13 (3),121-124.

3. Gomez H et al. (1996) Nutricion Hospitalaria, Spain 11 (2), 122-130.

4. Chan SL (2004) Annals of the Academy of Medicine, Singapore 33 (4), 494-498.

5. Ioannides-Demos, LL et al. (1995) Medical Journal of Australia 163 (5), 233, 235-237. 